\title{
浅谈守恒定律在分析化学教学中的应用
}

薛庆旺，贾丽萍，贾文丽，王术皓，李爱峰*

聊城大学化学化工学院, 山东 聊城 252000

摘要: 守恒是自然界普遍遵循的定律, 在分析化学中也涉及到许多守恒定律, 如物料守恒、电荷守恒、质子守恒、电子 守恒等。在分析化学教学中, 守恒定律不仅用于许多公式的推导, 还能用来解决复杂的计算问题。笔者在长期的教学实 践中发现运用守恒定律来解决问题, 不仅能帮助学生了解公式的来龙去脉, 还有助于帮助学生从宏观全局考虑问题, 将 复杂的过程简单化, 而不必在过程细节上纠缠。

关键词: 分析化学; 物料守恒; 电荷守恒; 质子守恒; 电子守恒

中图分类号: G64; O6

\section{A Tentative Analysis of the Application of Conservation Law in Analytical Chemistry Teaching}

Qingwang Xue, Liping Jia, Wenli Jia, Shuhao Wang, Aifeng Li *

College of Chemistry and Chemical Engineering, Liaocheng University, Liaocheng 252000, Shandong Province, China.

Abstract: Conservation law is widespread in nature. Analytical chemistry is often related to many conservation laws, such as material conservation, charge conservation, proton conservation, electron conservation, etc. In analytical chemistry, conservation laws can be used to derive many formulas and to solve some complex calculation problems. In long-term teaching practice, the writers find that conservation laws can help students to understand many formulas, simplify the complicated process, consider the problem on an overall perspective, and not to consider trivial details.

Key Words: Analytical chemistry; Material conservation; Charge conservation; Proton conservation; Electron conservation

守恒存在于整个自然界的千变万化之中, 一切化学反应都遵循守恒定律, 在化学变化中有各种 各样的守恒, 如质量守恒、能量守恒、元素守恒、电子守恒、电荷守恒等 ${ }^{[1]}$ 。在分析化学中也不例 外, 许多公式的推导过程要用到守恒定律。此外, 在有关化学平衡和滴定分析的计算中, 运用守恒 定律只需要考虑反应体系中某些组分相互作用前后某些物理量或化学量的始态和终态, 利用其中某 种不变的量建立关系式, 不必了解过多的中间过程, 避免了繁杂的分析和多重化学反应, 从而达到 速解、巧解化学习题的目的 ${ }^{[2]}$ 。笔者在长期的教学过程中发现这种 “纵览全局, 抓住守恒, 不看中 间, 只看两头” 的解题思路具有思路简单、关系明确、计算快捷、不易出错等优点, 更容易为学生 所接受 ${ }^{[3]}$ 。本文简要介绍守恒定律在分析化学教学中的应用。

收稿: 2021-04-06; 录用: 2021-05-03; 网络发表: 2021-05-17

“通讯作者, Email: 1xpsdta2001@sina.com

基金资助：聊城大学一流本科课程培育项目(Y201940); 聊城大学教学改革项目(G202021, G202060) 


\section{1 公式的推导}

\section{1 质子平衡式的列出方法}

在酸碱反应中酸失去质子, 碱得到质子, 因此, 酸碱反应的实质是质子的转移, 在酸碱转移质 子的过程中, 酸失去质子的总数与碱得到质子的总数必然相等, 即质子是守恒的。在酸碱溶液中, 有些物质(即酸)失去质子, 有些物质(即碱)得到质子, 因此, 得质子产物与失质子产物的浓度之间存 在一定的关系。根据质子守恒, 可以列出质子平衡式(proton balance equation, PBE)。

PBE的书写有两种方法: 推导法和直接列出法。前者是在物料平衡式(material balance equation, $\mathrm{MBE}$ )及电荷平衡式(charge balance equation, $\mathrm{CBE}$ )的基础上推导得出。下面以 $c \mathrm{~mol} \cdot \mathrm{L}^{-1} \mathrm{Na}_{2} \mathrm{CO}_{3}$ 溶液 为例, 说明PBE的推导过程。

首先列出 2 个MBE:

$$
\begin{aligned}
& {\left[\mathrm{Na}^{+}\right]=2 c} \\
& {\left[\mathrm{CO}_{3}^{2-}\right]+\left[\mathrm{HCO}_{3}^{-}\right]+\left[\mathrm{H}_{2} \mathrm{CO}_{3}\right]=2 c}
\end{aligned}
$$

再列出 $\mathrm{CBE}$ :

$$
\left[\mathrm{Na}^{+}\right]+\left[\mathrm{H}^{+}\right]=2\left[\mathrm{CO}_{3}^{2-}\right]+\left[\mathrm{HCO}_{3}^{-}\right]+\left[\mathrm{OH}^{-}\right]
$$

将 2 个 MBE代入CBE:

$$
2\left[\mathrm{CO}_{3}^{2-}\right]+2\left[\mathrm{HCO}_{3}^{-}\right]+2\left[\mathrm{H}_{2} \mathrm{CO}_{3}\right]+\left[\mathrm{H}^{+}\right]=2\left[\mathrm{CO}_{3}^{2-}\right]+\left[\mathrm{HCO}_{3}^{-}\right]+\left[\mathrm{OH}^{-}\right]
$$

整理可得PBE:

$$
\left[\mathrm{H}^{+}\right]=\left[\mathrm{OH}^{-}\right]-\left[\mathrm{HCO}_{3}^{-}\right]-2\left[\mathrm{H}_{2} \mathrm{CO}_{3}\right]
$$

如图1所示, 以 $\mathrm{Na}_{2} \mathrm{CO}_{3}$ 溶液为例, 说明直接列出法的步骤。

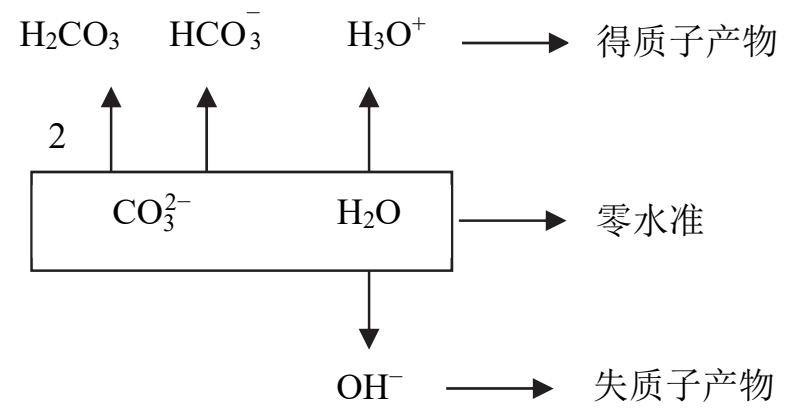

图1 $\mathrm{Na}_{2} \mathrm{CO}_{3}$ 溶液PBE的列出方法

(1) 选择溶液中大量存在并参与质子得失的物质作为零水准, 通常是起始的酸碱组分 $\left(\mathrm{CO}_{3}^{2-}\right)$ 及 溶剂 $\left(\mathrm{H}_{2} \mathrm{O}\right)$ 。

(2) 将溶液中其他的组分 $\left(\mathrm{HCO}_{3}^{-} 、 \mathrm{H}_{2} \mathrm{CO}_{3} 、 \mathrm{H}^{+} 、 \mathrm{OH}^{-}\right)$与零水准进行比较, 考查得失质子的情况。

(3) 直接列出 $\mathrm{PBE}\left(\left[\mathrm{H}^{+}\right]+\left[\mathrm{HCO}_{3}^{-}\right]+2\left[\mathrm{H}_{2} \mathrm{CO}_{3}\right]=\left[\mathrm{OH}^{-}\right]\right)$, 将得失质子的产物的浓度分别列在等号 的两侧, 得失质子的数目为相应浓度项的系数。

(4) 将列出的PBE进行整理 $\left(\left[\mathrm{H}^{+}\right]=\left[\mathrm{OH}^{-}\right]-\left[\mathrm{HCO}_{3}^{-}\right]-2\left[\mathrm{H}_{2} \mathrm{CO}_{3}\right]\right)$, 将 $\left[\mathrm{H}^{+}\right]$放在等号的左侧, 其余各 项整理至等号的右侧。

\section{2 分布分数的计算公式}

溶液中某酸碱组分的平衡浓度占其总浓度的分数, 称为分布分数, 以 $\delta$ 表示。 $\delta$ 将酸碱型体的平 衡浓度与酸碱的总浓度联系起来, 能定量说明溶液中各种酸碱组分的分布情况。通常酸碱的总浓度 已知, 知道了 $\delta$, 便可求得溶液中各种酸碱组分的平衡浓度, 这在酸碱平衡的学习中是十分重要的。 在 $\delta$ 的推导过程中, 也用到了物质守恒。下面, 以一元弱酸 HA 为例, 说明 $\delta$ 的推导过程: 首 
先, 列出平衡常数的表达式:

$$
K_{\mathrm{a}}=\frac{\left[\mathrm{H}^{+}\right]\left[\mathrm{A}^{-}\right]}{[\mathrm{HA}]}
$$

整理可得:

$$
\left[\mathrm{A}^{-}\right]=\frac{K_{\mathrm{a}}[\mathrm{HA}]}{\left[\mathrm{H}^{+}\right]}
$$

代入物料平衡式，可得:

$$
c=[\mathrm{HA}]+\left[\mathrm{A}^{-}\right]=[\mathrm{HA}]+\frac{K_{\mathrm{a}}[\mathrm{HA}]}{\left[\mathrm{H}^{+}\right]}=[\mathrm{HA}]\left(1+\frac{K_{\mathrm{a}}}{\left[\mathrm{H}^{+}\right]}\right)
$$

将整理得到的物料平衡式代入酸型体 $\delta$ 的定义中, 整理可得:

$$
\delta_{\mathrm{HA}}=\frac{[\mathrm{HA}]}{c}=\frac{1}{1+\frac{K_{\mathrm{a}}}{\left[\mathrm{H}^{+}\right]}}=\frac{\left[\mathrm{H}^{+}\right]}{\left[\mathrm{H}^{+}\right]+K_{\mathrm{a}}}
$$

根据物质守恒, 酸型体与碱型体的 $\delta$ 的加和等于 1 , 可得碱型体的 $\delta$ :

$$
\delta_{\mathrm{A}^{-}}=\frac{\left[\mathrm{A}^{-}\right]}{c}=1-\delta_{\mathrm{HA}}=\frac{K_{\mathrm{a}}}{\left[\mathrm{H}^{+}\right]+K_{\mathrm{a}}}
$$

\section{3 终点误差公式}

在酸碱滴定中, 由于酸碱指示剂的变色点与化学计量点不重合引起的误差称为滴定误差(titration error)或终点误差(ending point error), 用 $E_{\mathrm{t}}$ 或TE表示。 $E_{\mathrm{t}}$ 的推导也用到了 $\mathrm{MBE}$ 及 CBE。

下面以浓度为 $c\left(\mathrm{~mol} \cdot \mathrm{L}^{-1}\right)$ 的 $\mathrm{NaOH}$ 滴定浓度为 $c_{0}\left(\mathrm{~mol} \cdot \mathrm{L}^{-1}\right)$ 、体积为 $V_{0}(\mathrm{~mL})$ 的 $\mathrm{HCl}$ 溶液为例, 说明 $E_{\mathrm{t}}$ 的 推导过程: 设滴定终点时, 消耗 $\mathrm{NaOH}$ 溶液的体积为 $V(\mathrm{~mL})$ 。根据相对误差的定义, 终点误差可以表示 为:

$$
E_{\mathrm{t}}=\frac{c V-c_{0} V_{0}}{c_{0} V_{0}}
$$

因此 $E_{\mathrm{t}}$ 的正负及大小取决于 $c V$ 和 $c_{0} V_{0}$ 的相对大小。列出 2 个 $\mathrm{MBE}$ :

$$
\begin{aligned}
& {\left[\mathrm{Na}^{+}\right]^{\mathrm{ep}}=c_{\mathrm{NaOH}}^{\mathrm{ep}}=\frac{c V}{V+V_{0}}} \\
& {\left[\mathrm{Cl}^{-}\right]^{\mathrm{ep}}=c_{\mathrm{HCl}}^{\mathrm{ep}}=\frac{c_{0} V_{0}}{V+V_{0}}}
\end{aligned}
$$

列出 $\mathrm{CBE}$ :

$$
\left[\mathrm{Na}^{+}\right]^{\mathrm{ep}}+\left[\mathrm{H}^{+}\right]^{\mathrm{ep}}=\left[\mathrm{Cl}^{-}\right]^{\mathrm{ep}}+\left[\mathrm{OH}^{-}\right]^{\mathrm{ep}}
$$

将式(2)、(3)和(4)代入式(1), 整理可得:

$$
E_{\mathrm{t}}=\frac{\left[\mathrm{OH}^{-}\right]^{\mathrm{ep}}-\left[\mathrm{H}^{+}\right]^{\mathrm{ep}}}{c_{\mathrm{HCl}}^{\mathrm{ep}}}
$$

由式(5)可知, $E_{\mathrm{t}}$ 的大小与滴定终点时 $\mathrm{HCl}$ 溶液的浓度成反比, 浓度越大, 误差越小。由于滴定终 点时溶液的总体积与化学计量点时差距甚小, 因此滴定终点时 $\mathrm{HCl}$ 溶液的浓度与化学计量点时近似相 等, 式(5)也可写作:

$$
E_{\mathrm{t}}=\frac{\left[\mathrm{OH}^{-}\right]^{\mathrm{ep}}-\left[\mathrm{H}^{+}\right]^{\mathrm{ep}}}{c_{\mathrm{HCl}}^{\mathrm{sp}}}
$$

由上述推导过程可知, $E_{\mathrm{t}}$ 的计算公式是在定义式的基础上, 引入 $\mathrm{MBE}$ 及 $\mathrm{CBE}$ 后逐步推导得出的。 


\section{4 化学需氧量的计算公式}

化学需氧量又称COD (chemical oxygen demand), 是在水质分析中表征水体被微量有机物和无机 可氧化物质污染程度的常用指标, 它是将测定过程中消耗的氧化剂的量折合成氧气的质量浓度 $\left(\mathrm{mg} \cdot \mathrm{L}^{-1}\right)$ 来表示。如图2所示, 以酸性高锰酸钾法测定 COD为例分析测定步骤及计算公式的推导过程。

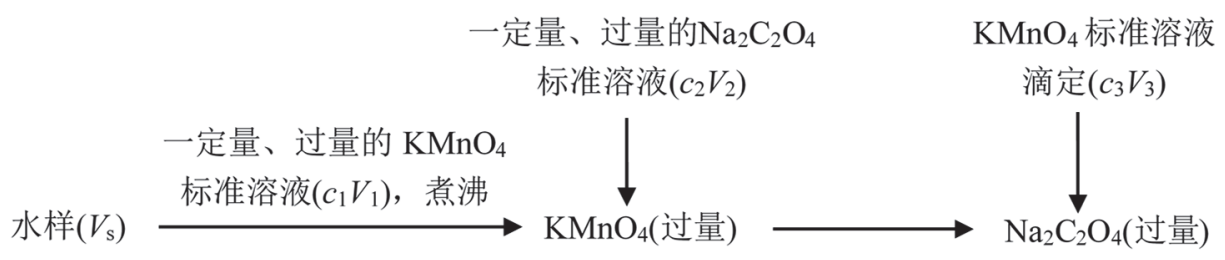

$$
\mathrm{COD}_{\mathrm{Mn} \text { (酸性) }}=\frac{\frac{5}{4}\left(c_{1} V_{1}+c_{3} V_{3}-\frac{2}{5} c_{2} V_{2}\right) M_{\mathrm{O}_{2}}}{V_{\mathrm{s}}}\left(\mathrm{mg} \cdot \mathrm{L}^{-1}\right)
$$

图2 酸性锰酸钾法测定COD的实验步骤及计算公式

根据上述测定步骤, 可知: 先后总共向水样中加入氧化剂 $\mathrm{KMnO}_{4}$ 的物质的量为 $c_{1} V_{1}+c_{3} V_{3}$, 与其 发生反应的还原剂有污染物和 $\mathrm{Na}_{2} \mathrm{C}_{2} \mathrm{O}_{4}$ 。氧化剂的量是守恒的, 即污染物消耗的 $\mathrm{KMnO}_{4}$ 的物质的量 与 $\mathrm{Na}_{2} \mathrm{C}_{2} \mathrm{O}_{4}$ 消耗的 $\mathrm{KMnO}_{4}$ 的物质的量之和等于 $\mathrm{KMnO}_{4}$ 的总物质的量。 $\mathrm{Na}_{2} \mathrm{C}_{2} \mathrm{O}_{4}$ 消耗的 $\mathrm{KMnO}_{4}$ 的物质 的量为 $2 / 5 c_{2} V_{2}$ 。因此, 污染物消耗的 $\mathrm{KMnO}_{4}$ 的物质的量为 $c_{1} V_{1}+c_{3} V_{3}-2 / 5 c_{2} V_{2}$ 。由于在酸性高锰酸 钾法中, $1 \mathrm{~mol} \mathrm{KMnO}_{4}$ 得 $5 \mathrm{~mol}$ 电子还原为 $\mathrm{Mn}^{2+}$ 。如果用 $\mathrm{O}_{2}$ 做氧化剂, $1 \mathrm{~mol} \mathrm{O}_{2}$ 得 $4 \mathrm{~mol}$ 电子还原为 $\mathrm{H}_{2} \mathrm{O}$ 。 因此, $1 \mathrm{~mol} \mathrm{KMnO}_{4}$ 的氧化能力相当于 $5 / 4 \mathrm{~mol} \mathrm{O}_{2},\left(c_{1} V_{1}+c_{3} V_{3}-2 / 5 c_{2} V_{2}\right) \times 5 / 4$ 就是污染物消耗的 $\mathrm{O}_{2}$ 的物质的量。这样, COD的计算公式就不难理解了。

\section{2 守恒定律在分析化学计算中的应用}

利用守恒定律解决化学计算的问题是抓住物质变化过程中某一特定的量固定不变来列式求 解, 关键是抓住其中的守恒关系。在有关化学平衡和滴定分析的计算中经常会用到守恒定律, 如元 素守恒、电子守恒等, 下面结合例题说明守恒定律的妙用。

\section{1 元素守恒}

例 ${ }^{[4]}$ : 称取含 $\mathrm{Ni}$ 的试样 $0.2000 \mathrm{~g}$, 溶解后加入 $5.00 \mathrm{~mL} 0.02000 \mathrm{~mol} \cdot \mathrm{L}^{-1} \mathrm{AgNO}_{3}$ 为指示剂, 加入过 量氨水和 $\mathrm{KI}$, 用 $0.05000 \mathrm{~mol} \cdot \mathrm{L}^{-1} \mathrm{KCN}$ 标准溶液滴定至 $\mathrm{AgI}$ 沉淀全部消失即为终点, 用去 $24.40 \mathrm{~mL}$, 已 知滴定反应的产物为 $\mathrm{Ni}\left(\mathrm{CN}^{-}\right)_{4}^{2-}$ 和 $\mathrm{Ag}\left(\mathrm{CN}^{-}\right)_{2}^{-}$, 计算试样中 $w_{\mathrm{Ni}}$ (已知 $A_{\mathrm{Ni}}=58.69$ )。

解：由于滴定反应的产物为 $\mathrm{Ni}\left(\mathrm{CN}^{-}\right)_{4}^{2-}$ 和 $\mathrm{Ag}\left(\mathrm{CN}^{-}\right)_{2}^{-}$, 根据 $\mathrm{CN}^{-}$的物质的量守恒, 可得:

$$
n_{\mathrm{CN}^{-}}=4 n_{\mathrm{Ni}^{2+}}+2 n_{\mathrm{Ag}^{+}}
$$

代入数据, 可得:

$$
\begin{aligned}
& n_{\mathrm{Ni}^{2+}}=\frac{n_{\mathrm{CN}^{-}}-2 n_{\mathrm{Ag}^{+}}}{4}=\frac{0.05000 \times 24.40-2 \times 0.02000 \times 5.00}{4}=0.255(\mathrm{mmol}) \\
& w_{\mathrm{Ni}^{2+}}=\frac{n_{\mathrm{Ni}^{2+}} A_{\mathrm{Ni}}}{m_{\mathrm{s}}}=\frac{0.255 \times 10^{-3} \times 58.69}{0.2000} \times 100 \%=7.48 \%
\end{aligned}
$$

由于现有的分析化学教材在配位滴定法一章中重点介绍EDTA为滴定剂的配位滴定法, 仅仅简单介 绍录量法和氰量法。因此, 学生往往对于氰量法测定 Ni的原理不太清楚, 遇到上述习题感到不知所措。 只要搞清楚Ni元素的最终存在形式是 $\mathrm{Ni}\left(\mathrm{CN}^{-}\right)_{4}^{2-}$ 和 $\mathrm{Ag}\left(\mathrm{CN}^{-}\right)_{2}^{-}$, 利用元素守恒列出等量关系就能使问题 迎刃而解了。 


\section{2 电子守恒}

例 $2^{[4]}$ : 移取乙二醇试液 $25.00 \mathrm{~mL}$, 加入 $0.04500 \mathrm{~mol} \cdot \mathrm{L}^{-1} \mathrm{KMnO}_{4}$ 的碱性溶液 $50.00 \mathrm{~mL}$, 反应完全后, 酸化溶液, 加入 $0.3000 \mathrm{~mol} \cdot \mathrm{L}^{-1} \mathrm{Na}_{2} \mathrm{C}_{2} \mathrm{O}_{4}$ 溶液 $10.00 \mathrm{~mL}$, 此时所有高价锰均还原至 $\mathrm{Mn}^{2+}$, 以 $0.04500 \mathrm{~mol} \cdot \mathrm{L}^{-1}$ $\mathrm{KMnO}_{4}$ 溶液滴定过量的 $\mathrm{Na}_{2} \mathrm{C}_{2} \mathrm{O}_{4}$, 终点时消耗 $2.30 \mathrm{~mL}$, 求试液中乙二醇的质量。 $\left(M r\left(\mathrm{HOC}_{2} \mathrm{H}_{4} \mathrm{OH}\right)=62.05\right)$

解法1：设 $25.00 \mathrm{~mL}$ 乙二醇试液中含乙二醇 $x \mathrm{mmol}$ 。

乙二醇与 $\mathrm{KMnO}_{4}$ 的反应:

$$
\mathrm{HOC}_{2} \mathrm{H}_{4} \mathrm{OH}+10 \mathrm{MnO}_{4}^{-}+14 \mathrm{OH}^{-}=2 \mathrm{CO}_{3}^{2-}+10 \mathrm{MnO}_{4}^{2-}+10 \mathrm{H}_{2} \mathrm{O}
$$

$$
10 x
$$

酸化后: $3 \mathrm{MnO}_{4}^{2-}+4 \mathrm{H}^{+}=2 \mathrm{MnO}_{4}^{-}+\mathrm{MnO}_{2} \downarrow+2 \mathrm{H}_{2} \mathrm{O}$

$$
10 x \quad 20 x / 3 \quad 10 x / 3
$$

加入 $\mathrm{Na}_{2} \mathrm{C}_{2} \mathrm{O}_{4}$ 后:

$$
\begin{gathered}
2 \mathrm{MnO}_{4}^{-}+16 \mathrm{H}^{+}+5 \mathrm{C}_{2} \mathrm{O}_{4}^{2-}=2 \mathrm{Mn}^{2+}+10 \mathrm{CO}_{2} \uparrow+8 \mathrm{H}_{2} \mathrm{O} \\
0.04500 \times 50.00-10 x+20 x / 3 \quad(0.04500 \times 50.00-10 x+20 x / 3) \times 2.5 \\
\mathrm{MnO}_{2}+4 \mathrm{H}^{+}+\mathrm{C}_{2} \mathrm{O}_{4}^{2-}=\mathrm{Mn}^{2+}+2 \mathrm{CO}_{2} \uparrow+2 \mathrm{H}_{2} \mathrm{O} \\
10 x / 3 \quad 10 x / 3
\end{gathered}
$$

滴定过程中: $2 \mathrm{MnO}_{4}^{-}+16 \mathrm{H}^{+}+5 \mathrm{C}_{2} \mathrm{O}_{4}^{2-}=2 \mathrm{Mn}^{2+}+10 \mathrm{CO}_{2} \uparrow+8 \mathrm{H}_{2} \mathrm{O}$

剩余 $\mathrm{Na}_{2} \mathrm{C}_{2} \mathrm{O}_{4}$ 的量为: $[0.3000 \times 10.00-(0.04500 \times 50.00-10 x+20 x / 3) \times 2.5-10 x / 3] \mathrm{mmol}$

剩余的 $\mathrm{Na}_{2} \mathrm{C}_{2} \mathrm{O}_{4}$ 刚好与 $2.30 \mathrm{~mL} 0.04500 \mathrm{~mol} \cdot \mathrm{L}^{-1} \mathrm{KMnO}_{4}$ 溶液完全反应,

因此: $0.3000 \times 10.00-(0.04500 \times 50.00-10 x+20 x / 3) \times 2.5-10 x / 3=2.30 \times 0.04500 \times 2.5$

解得: $x=0.5768 \mathrm{mmol}$

因此: $m_{\mathrm{HOC}_{2} \mathrm{H}_{4} \mathrm{OH}}=x M \mathrm{r}=0.5768 \times 62.05=35.79 \mathrm{mg}$

解法 2: 首先, 根据参与氧化还原反应的物质的最终产物, 分析得失电子的数目:

$$
\mathrm{MnO}_{4}^{-} \stackrel{+5 \mathrm{e}}{\longrightarrow} \mathrm{Mn}^{2+} \quad \mathrm{C}_{2} \mathrm{O}_{4}^{2-} \stackrel{-2 \mathrm{e}}{\longrightarrow} 2 \mathrm{CO}_{2} \quad \mathrm{HOC}_{2} \mathrm{H}_{4} \mathrm{OH} \stackrel{-10 \mathrm{e}}{\longrightarrow} 2 \mathrm{CO}_{3}^{2-}
$$

根据电子守恒, 可得: $2 n_{\mathrm{Na}_{2} \mathrm{C}_{2} \mathrm{O}_{4}}+10 n_{\mathrm{HOC}_{2} \mathrm{H}_{4} \mathrm{OH}}=5 n_{\mathrm{KMnO}_{4}}$

整理, 可得: $n_{\mathrm{HOC}_{2} \mathrm{H}_{4} \mathrm{OH}}=\frac{5 n_{\mathrm{KMnO}_{4}}-2 n_{\mathrm{Na}_{2} \mathrm{C}_{2} \mathrm{O}_{4}}}{10}$

代入数据, 可得: $m_{\mathrm{HOC}_{2} \mathrm{H}_{4} \mathrm{OH}}=\frac{5 \times 0.04500 \times(50.00+2.30)-2 \times 0.3000 \times 10.00}{10}=0.5768 \mathrm{mmol}$

$$
m_{\mathrm{HOC}_{2} \mathrm{H}_{4} \mathrm{OH}}=x M \mathrm{r}=0.5768 \times 62.05=35.79 \mathrm{mg}
$$

通过比较 2 种解法可以看出: 解法 1 是将题目涉及到的每一个氧化还原反应进行剖析, 搞清楚反 应物及生成物的物质的量, 步骤繁琐而且容易出错。解法2没有考虑中间的细节, 而是搞清楚氧化剂 及还原剂的始态和终态, 分析得失电子的数目, 根据电子守恒直接列出等量关系式, 步骤简单、清 晰易懂且不易出错。

\section{3 元素守恒与电子守恒并用}

例 ${ }^{[5]}$ : 已知 $\mathrm{I}_{2}$ 在水中的溶解度为 $0.00133 \mathrm{~mol} \cdot \mathrm{L}^{-1}$, 求以 $0.05000 \mathrm{~mol} \cdot \mathrm{L}^{-1} \mathrm{Ce}^{4+}$ 滴定 $50.00 \mathrm{~mL}$ 等浓 度的 $\mathrm{I}^{-}$时, 固体 $\mathrm{I}_{2}$ 刚刚出现沉淀时, 消耗的 $\mathrm{Ce}^{4+}$ 为多少毫升? (反应: $\mathrm{I}_{2}+\mathrm{I}^{-} \rightarrow \mathrm{I}_{3}^{-}$的 $K=708$ )

解法 1: 设消耗的 $\mathrm{Ce}^{4+}$ 为 $x$ 毫升

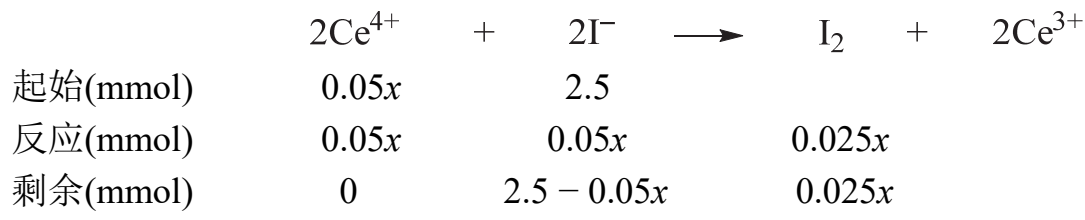




$\begin{array}{lcccc} & \mathrm{I}_{2} & + & \mathrm{I}^{-} & \mathrm{I}_{3}^{-} \\ \text {起始 }(\mathrm{mmol}) & 0.025 x & 2.5-0.05 x & 0 \\ \text { 剩余 }(\mathrm{mmol}) & 0.00133(50+x) & 2.5-0.075 x+0.00133(50+x) & & 0.025 x-0.00133(50+x) \\ \text { 反应 }(\mathrm{mmol}) & 0.025 x-0.00133(50+x) & 0.025 x-0.00133(50+x) & & 0.025 x-0.00133(50+x)\end{array}$

因此, 可以将 $\mathrm{I}_{2} 、 \mathrm{I}^{-} 、 \mathrm{I}_{3}^{-}$的平衡浓度表示为:

$$
\begin{aligned}
& {\left[\mathrm{I}_{2}\right]=0.00133 \mathrm{~mol} \cdot \mathrm{L}^{-1}} \\
& {\left[\mathrm{I}^{-}\right]=\frac{n_{\mathrm{I}^{-}}}{V}=\frac{2.5-0.075 x+0.00133 \times(50+x)}{50+x}=\frac{2.5665-0.07367 x}{50+x} \mathrm{~mol} \cdot \mathrm{L}^{-1}} \\
& {\left[\mathrm{I}_{3}^{-}\right]=\frac{n_{\mathrm{I}_{3}^{-}}}{V}=\frac{0.025 x-0.00133 \times(50+x)}{50+x}=\frac{0.0237 x-0.065}{50+x} \mathrm{~mol} \cdot \mathrm{L}^{-1}}
\end{aligned}
$$

将上述 3 个式子代入平衡常数的表达式, 可得:

$$
\begin{aligned}
& K=\frac{\left[\mathrm{I}_{3}^{-}\right]}{\left[\mathrm{I}^{-}\right]\left[\mathrm{I}_{2}\right]}=\frac{0.0237 x-0.065}{50+x} \times \frac{1}{0.00133} \times \frac{50+x}{2.5665-0.07367 x} \\
& =\frac{0.0237 x-0.065}{0.00133 \times(2.5665-0.07367 x)} \\
& =708
\end{aligned}
$$

解得: $x=26.67 \mathrm{~mL}$

解法 2: 设消耗的 $\mathrm{Ce}^{4+}$ 为 $x$ 毫升, 固体 $\mathrm{I}_{2}$ 刚刚出现沉淀时, 溶液的总体积为 $V$ 毫升 $(V=50+x)$ 。首先, 分析氧化剂与还原剂得失电子的数目:

根据电子守恒, 可以列出:

$$
\begin{aligned}
& \mathrm{Ce}^{4+} \stackrel{+\mathrm{e}}{\longrightarrow} \mathrm{Ce}^{3+} \quad 2 \mathrm{I}^{-} \stackrel{-2 \mathrm{e}}{\longrightarrow} \mathrm{I}_{2} \\
& n_{\mathrm{Ce}^{4+}}=2 \times\left(n_{\mathrm{I}_{2}}+n_{\mathrm{I}_{3}^{-}}\right)=2 \times\left(\left[\mathrm{I}_{2}\right]+\left[\mathrm{I}_{3}^{-}\right]\right) V
\end{aligned}
$$

整理可得:

$$
\left[\mathrm{I}_{2}\right]+\left[\mathrm{I}_{3}^{-}\right]=0.00133+\left[\mathrm{I}_{3}^{-}\right]=\frac{n_{\mathrm{Ce}^{4+}}}{2 V}=\frac{0.05 x}{2 \times(50+x)}=\frac{0.025 x}{50+x}
$$

则:

$$
\left[\mathrm{I}_{3}^{-}\right]=\frac{0.025 x}{50+x}-0.00133
$$

由于I有 3 种存在型体 $\left(\mathrm{I}_{2} 、 \mathrm{I}^{-} 、 \mathrm{I}_{3}^{-}\right)$, 根据I的物质的量守恒, 可以列出:

$$
n_{\mathrm{I}^{-}}=\left(3 \times\left[\mathrm{I}_{3}^{-}\right]+2 \times\left[\mathrm{I}_{2}\right]+\left[\mathrm{I}^{-}\right]\right) V
$$

代入数据, 可得:

$$
0.05 \times 50=\left(3 \times\left[\mathrm{I}_{3}^{-}\right]+2 \times 0.00133+\left[\mathrm{I}^{-}\right]\right) \times(50+x)
$$

整理可得:

$$
\begin{aligned}
{\left[\mathrm{I}^{-}\right] } & =\frac{0.05 \times 50}{50+x}-2 \times 0.00133-3 \times\left[\mathrm{I}_{3}^{-}\right] \\
& =\frac{0.05 \times 50}{50+x}-2 \times 0.00133-3 \times\left(\frac{0.025 x}{50+x}-0.00133\right) \\
& =\frac{2.5-0.075 x}{50+x}+0.00133
\end{aligned}
$$

将(7)、(8)式代入平衡常数的定义式, 可得: 


$$
K=\frac{\left[\mathrm{I}_{3}^{-}\right]}{\left[\mathrm{I}_{2}\right]\left[\mathrm{I}^{-}\right]}=\frac{\frac{0.025 x}{50+x}-0.00133}{0.00133 \times\left(\frac{2.5-0.075 x}{50+x}+0.00133\right)}=708
$$

解得: $x=26.67 \mathrm{~mL}$

与例 2 相比, 例 3 虽然涉及到的氧化还原反应的个数较少, 但还涉及到络合平衡, 因此情况变得更 加复杂, 只要抓住氧化还原反应中的电子守恒及络合平衡中的元素守恒, 就能列出等量关系式, 达到 速解、巧解的目的。

\section{3 结语}

综上所述, 在分析化学教学中, 运用守恒定律推导公式可以帮助学生了解公式的来龙去脉, 更 好地利用公式解决问题; 运用守恒定律解决复杂的计算问题, 特别是氧化还原滴定的相关计算, 不 仅能够速解、巧解问题, 更重要的是帮助学生从宏观全局的视角思考问题, 形成科学的思维方式, 从整体上把握问题的关键, 从复杂的内容中理清头绪, 将复杂的过程简单化, 而不是在繁琐的过程 细节上过多地纠缠 ${ }^{[6]}$ 。守恒定律的巧妙运用要求教师具有渊博的知识、丰富的教学经验及高超的总 揽全局的能力。只要教师认真钻研教学内容, 仔细揣摩分析解决问题的思路, 发现解决问题的切入 点, 就可以灵活地运用这种 “重过程、巧守恒”的教学策略取得良好的教学效果 ${ }^{[7]}$ 。

\section{参 考 文 献}

[1] 张珊珊. 课程教育研究, 2015, No. 5, 183.

[2] 孙本义. 学周刊, 2011, No. 2, 145 .

[3] 谢勿玉, 衷明华. 江西化工, 2014, No. 1, 273.

[4] 武汉大学化学系分析化学教研室. 分析化学习题与例题. 北京: 高等教育出版社, 1999.

[5] 武汉大学. 分析化学. 第4版. 北京: 高等教育出版社, 2004: 263, 习题19.

[6] 万红霞. 学周刊, 2013, No. 12, 200.

[7] 诸全头. 化学教学, 2014, No. 9, 80 . 